\title{
Species Names Homonymy in Ladybirds (Coleoptera: Coccinellidae): 3
}

\author{
A. S. Ukrainsky \\ Department of Entomology, Biological Faculty, Moscow State University, Moscow, 119991 Russia \\ e-mail: para@proc.ru \\ Received December 9, 2008
}

Key words: Coleoptera, Coccinellidae, fossil, ladybirds, Coccinella, Chilocorus, nomenclature.

DOI: $10.1134 / \mathrm{S} 0031030110010156$

The study of the nomenclature of fossil ladybird beetles (Coleoptera: Coccinellidae) revealed three previously overlooked cases of homonymy. Three species names proved to be preoccupied among recent species of the same family. According to the rules of the International Code of Zoological Nomenclature (1999, Articles 52, 53.3, 57, and 60), new names are proposed for junior primary homonyms having no synonyms.

\section{Coccinella ponomarenkoi Ukrainsky, nom. nov.}

Coccinella spectabilis Heer, 1865, p. 371, nom. praeocc. (non Coccinella spectabilis Faldermann, 1835, p. 450); Heer, 1876, p. 26; Handlirsch, 1906, p. 775.

Ety mology. The species is named in honor of the well-known Russian paleoentomologist Aleksandr Georgievich Ponomarenko.

\section{Coccinella heeri Ukrainsky, nom. nov.}

Coccinella decempustulata Heer, 1879, p. 397, nom. praeocc. (non Coccinella 10-pustulata Linnaeus, 1758, p. 368); Handlirsch, 1906 , p. 775.

Ety mology. The species is named in honor of Prof. Oswald Heer, the author of the original species name.

Chilocorus foersteri Ukrainsky, nom. nov.

Chilocorus politus Förster, 1891, p. 420, nom. praeocc. (non Chilocorus politus Mulsant, 1850, p. 455, 1033); Handlirsch, 1906, p. 776; SalomonCalvi, 1931, p. 84.

Ety mology. The species is named in honor of Bruno Förster, the author of the original species name.

\section{REFERENCES}

1. F. Faldermann, "Coleopterorum ab ill. Bungio in China boreali, Mongolia et montibus Altaicis collectorum, nee non ab ill. Turczaninoffio et Stschukino e provincia Irkutzk missorum illustrationes," Mem. Acad. Imp. Sci. St. Petersb. 2, 337-464 (1835).

2. B. Förster, "Die Insekten des "Plattigen Steinmergels" von Brunstatt," Abh. Geol. Specialkarte Elsass-Lothringen. Strassburg: R. Schultz. 3, 333-594 (1891).

3. A. Handlirsch, "Die fossilen Insekten und die Phylogenie der rezenten Formen," in Ein Handbuch für Paläontologen und Zoologen (Wilhelm Engelmann, Leipzig, 1906).

4. O. Heer, "Die Urwelt der Schweiz," (Friedrich Schulthess, Zürich, 1865).

5. O. Heer, The Primaeval World of Switzerland with 560 Illustrations (Longmans, Green and Co, London, 1876), Vol. 2.

6. O. Heer, "Die Urwelt der Schweiz," 2nd ed. (Friedrich Schulthess, Zürich, 1879).

7. International Code of Zoological Nomenclature, 4th ed. (Int. Trust Zool. Nomencl., London, 1999).

8. C. Linnaeus, Systema Naturae per regna tria Naturae, secundum classes, ordines, genera, species, cum Characteribus, Differentiis, Synonymis, Locis, 10th ed. (Stockholm, 1758), Vol. I.

9. M. É. Mulsant, "Species des coléoptères trimères sécuripalpes," Ann. Sci. Phys. Natur. Lyon, Agric. Industr. Lyon et Paris, Sér. 22 , XV+ 1-1104 (1850).

10. W. Salomon-Calvi (Ed.), Oberrheinischer Fossilkatalog (Gebruder Borntraeger, Berlin, 1931). 